\title{
Study of the Interference Affecting the Performance of the Theremin
}

\section{Carmen Bachiller Martín, Jorge Sastre Martínez, Amelia Ricchiuti, Héctor Esteban González, and Carlos Hernández Franco}

Universitat Politècnica de València, Camino de Vera s/n, 46022 València, Spain

Correspondence should be addressed to Carlos Hernández Franco, chernan@dcom.upv.es

Received 3 October 2011; Accepted 14 December 2011

Academic Editor: Miguel Ferrando

Copyright (c) 2012 Carmen Bachiller Martín et al. This is an open access article distributed under the Creative Commons Attribution License, which permits unrestricted use, distribution, and reproduction in any medium, provided the original work is properly cited.

The theremin is one of the earliest electronic musical instruments. It is named after the Russian physics Professor Lev S. Termen who invented it in 1919. This musical instrument belongs to a very short list of devices which are played without physical contact between the musician and the instrument. theremin players complain about the interference that any object in a radius of approximately 3 meters produces when playing the theremin, modifying the intonation of the instrument. This is a problem when playing in small scenarios, with other musicians which move around it. With the aim of reducing the degree of interference from nearby obstacles, some metallic isolating bars conforming an antenna array can be placed around the theremin pitch antenna. The paper shows different simulations calculated with the commercial software Ansoft HFSS, a tool which allows three-dimensional full wave electromagnetic field simulation, with radio frequencies, millimeter and micro waves, and experimental measures, both showing a reduction in the effect of the interference.

\section{Introduction}

The theremin is one of the earliest electronic musical instruments [1]. It is named after the Russian physics Professor Lev S. Termen who invented it in 1919. This musical instrument belongs to a very short list of devices which are played without physical contact between the musician and the instrument. That is the fascination and the special feature of this device which even today has many fans and supporters (see the "Theremin World" website [2]). It uses two antennas, one for the frequency or pitch control and one for the volume or dynamic control of the musical note produced. The musician can change both parameters by moving his/her hands around the antennas. In this case, the electromagnetic field around the antennas will be changed and be recognized by the hardware immediately.

The operation of the theremin is based on the oscillation in a resonant circuit and the superheterodyne principle. There are two oscillators in the circuitry that generate and control the frequency of the theremin signal; one of them is a fixed oscillator while the other one is variable. The frequency of the variable oscillator is controlled by the movement of one of the musician's hands. When the hand is moving near the antenna, the input impedance of the antenna changes provoking a change in the frequency of the variable oscillator.

When the hand is approaching, the frequency is increasing, and when the hand is moving away, the frequency is decreasing. Figure 1 summarizes a proposal of theremin block diagram.

The signal from the variable oscillator is mixed with the signal from the fixed oscillator. Then the result is filtered. In this way an acoustic signal can be generated whose pitch varies with the movements of the musician hand. The circuitry that controls the volume of the signal is also composed by two oscillators, one fixed and the other one variable.

When the musician hand is approaching or moving away from the volume antenna, a similar change on the input 


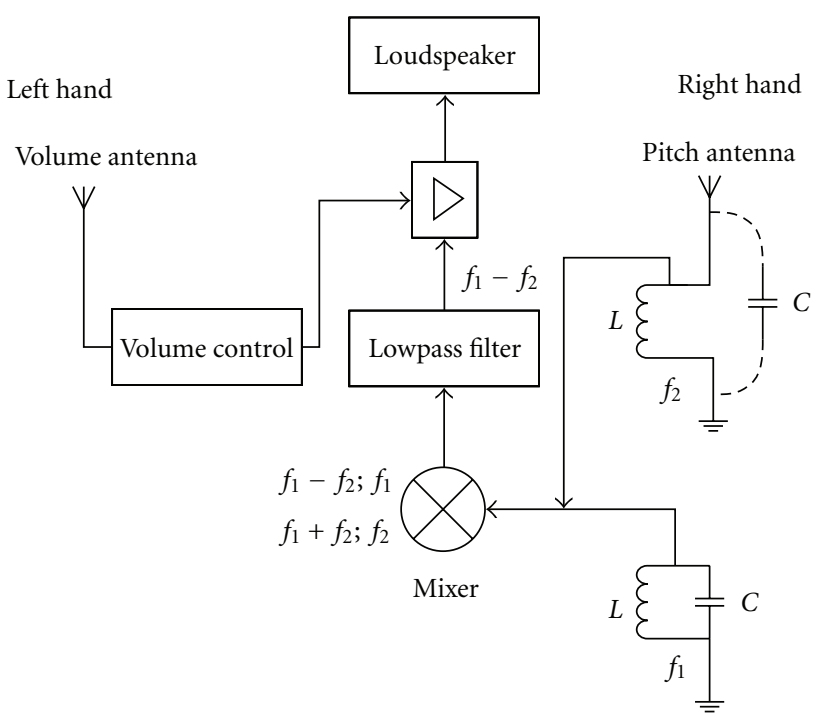

FIgURE 1: The theremin block diagram.

impedance of the antenna occurs, thus provoking a change on the frequency of the variable oscillator. If this variable frequency matches with the frequency of the fixed oscillator, then the output signal is maximized, thus producing maximum volume of the acoustic signal; otherwise the signal is lower.

The movements of both hands do not interfere with each other because both antennas (monopole for controlling the frequency and loop antenna for controlling the volume) produce electric fields with orthogonal polarizations.

\section{Theremin Drawbacks}

Since the appearance of the first theremins to nowadays different technologies has been used to implement the circuitry of the instrument. Nevertheless, the operation principle has been always the same.

This means that the techniques for playing the theremin and the difficulties that are associated with its operation are the same now as in 1920: the theremin is difficult to play since there is no space reference, it changes in each scenario and any object moving near the theremin interferes and makes the musician out of tune.

Moreover, theremins present the problem of nonlinear operation, since the distance between two consecutive notes is different in each octave.

The work of solving the nonlinearity of the theremin was undertaken by Lev Termen. He developed a theremin for Clara Rockmore that had a near linear behaviour [1]. Robert Moog in 2000 also developed the Etherwave Pro, with behaviour near the one of the Clara's theremin. Moreover, nowadays Thierry Frenkel is working on the improvement of volume response (http://theremin.tfrenkel.com/) [3]. Unfortunately, the study of the effect of the environment and the interference has been less studied. Buller and

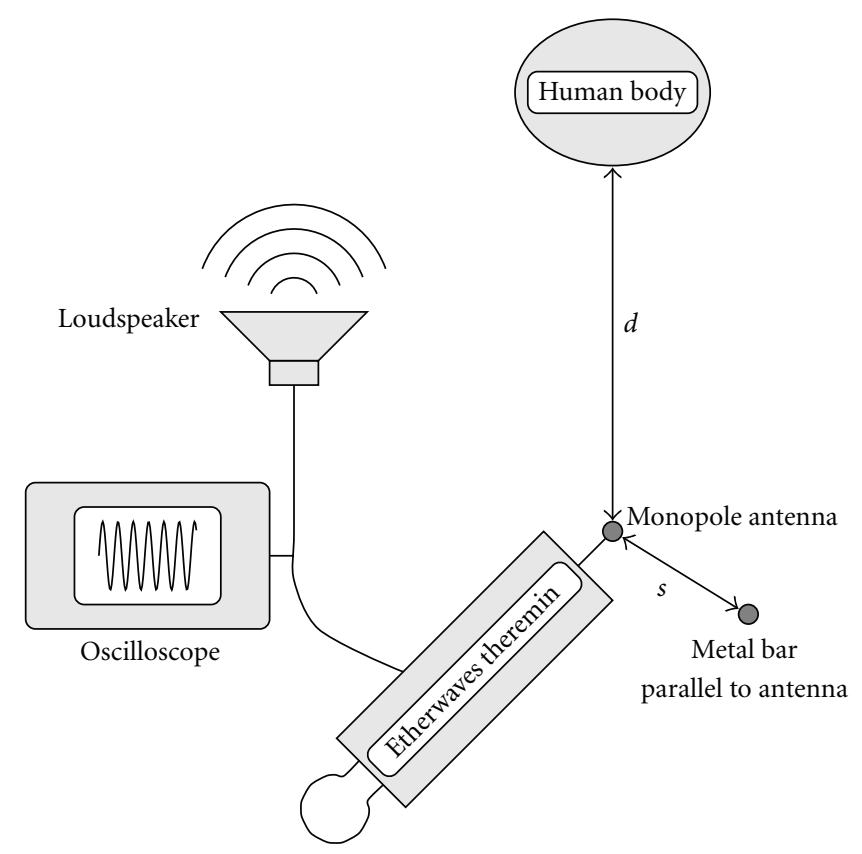

FIgURE 2: Measurement setup layout.

Wilson did some research concerning interference during the development of a position sensor based on the theremin principles [4].

\section{Theremin and Interferences}

Theremin players complain about the interference that any object in a radius of approximately $3 \mathrm{~m}$ produces when playing the theremin, modifying the intonation of the instrument [5]. This is a problem when playing in small scenarios, with other musicians which move around it.

Electromagnetic field radiation patterns of the theremin antennas are omnidirectional. The right antenna is used to control the pitch with horizontal movements of the hand. Any object moving near the theremin will alter the pitch, making the music out of tune. The left antenna controls the dynamics (volume) of the sound, with vertical movements of the left hand, and thus it is more difficult for moving objects to produce annoying effects on the music as with the right antenna. This paper presents a study to understand and minimize the effect of the environment in the theremin operation.

\section{How an Obstacle Interferes on the Theremin Operation}

4.1. Measurement Setup. In order to measure how a human presence interferes with the pitch of the theremin, the setup depicted in Figure 2 was prepared. A 2 meters long vertical metal bar was positioned parallel to and at a distance $s$ away from the theremin monopole antenna that controls the pitch. This metal bar takes the role of the hand of the musician, so 
that the distance s controls the desired intonation, with the advantage that the metal bar does not move and maintains the same intonation constant along time.

The output of the theremin is connected to an oscilloscope and to a loudspeaker. The oscilloscope is used to measure the amplitude and frequency of the output sound (which can be heard at the same time thanks to the loudspeaker). Finally, a person facing the antenna of the theremin approaches and moves away from it so that the distance from the person to the theremin monopole antenna d varies from $3 \mathrm{~m}$ to $60 \mathrm{~cm}$, measured from the lowest part of the theremin antenna to the waist of the human body at the same height. The human body weighted $60 \mathrm{Kg}$ and was $160 \mathrm{cms}$ tall.

The degree of interference of the human body is then measured in the following way: first no human body is placed near the theremin, and the distance $s$ from the theremin antenna to the metal bar that simulates the musician is adjusted to the first note $\mathrm{C}_{n}$ of the octave $\mathbf{n}$ under study (the susceptibility to interference in 6 octaves has been measured). The correct note is fixed by measuring the frequency with the oscilloscope and varying the metal bar position until the desired output frequency is achieved.

Next the interfering person is placed $3.05 \mathrm{~m}$ away from the theremin and the output frequency is measured with the oscilloscope at a given set of distances of that person starting from $3.05 \mathrm{~m}$ and ending at $60 \mathrm{~cm}$. Figure 3 presents the frequency variation in terms of musical notes in the twelve-tone equal temperament system (12-TET) versus the interfering human body distance in meters, for notes $\mathrm{C}_{2}, \mathrm{C}_{3}, \ldots, \mathrm{C}_{7}$.

The horizontal line in these figures indicates an out of tuning of a quarter tone higher than the original note, considering the 24-TET. In the higher pitched notes, the musician hand is so near the theremin antenna that the interference effect is quite small and only affects if the interfering human body is really very near the antenna.

On the other hand, the note frequencies are more different in the high-pitched area and this contributes also to reduce the effect of interference. In the middle-pitched area, the interference produces a higher tune of a quarter tone in the original note for distances so near as approximately $1.249 \mathrm{~m}$ for $\mathrm{C}_{4}$ and $1.05 \mathrm{~m}$ for $\mathrm{C}_{5}$.

Finally, for the lower-pitched area, those distances increase to approximately $1.665 \mathrm{~m}$ for $\mathrm{C}_{3}$ and $2.359 \mathrm{~m}$ for $\mathrm{C}_{2}$, because of the greater distance of the musician hand to the antenna to produce these notes and the lower frequency differences between musical notes in that area. All those distance values have been obtained applying linear interpolation between the measured values, and clearly they support the complaints of theremin players about the interferences that nearby objects produce in the theremin tune.

\section{A Proposal for Improvement}

5.1. Theoretical Analysis. With the aim of reducing the degree of interference from nearby obstacles, some metallic isolating bars conforming an antenna array can be placed around the theremin pitch antenna. In this section, we show different simulations calculated with the commercial software Ansoft HFSS, a tool which allows three-dimensional full wave electromagnetic field simulation, with radio frequencies, millimeter, and microwaves.

Before proceeding with the simulation of the antenna array, it was necessary to model in HFSS the theremin antenna which controls the pitch, that is, the monopole, which is a cylinder with a height of $462.6 \mathrm{~mm}$ and a radius of $4.7 \mathrm{~mm}$. The model of this antenna in HFSS was intended to be as close as possible to a real theremin monopole, but we found some difficulties.

In the first place, the theremin works at a frequency of $260 \mathrm{kHz}$, whilst the typical working frequency of HFSS is of the order of GHz. This does not mean that it is not possible to simulate at low frequency, but there are some limitations that must be taken into account. At $260 \mathrm{kHz}$, the wavelength is approximately $1,15 \mathrm{~km}$. At that frequency if we try to connect the monopole with a cable smaller than $0.9 \mathrm{~mm}$, the software gives an error because the electrical dimensions are too small, and the simulation can therefore not be finished.

Moreover, the antenna needs to be placed into a radiation box, with absorbent boundary conditions. Inside this box, the near field is calculated and then from that the far field. The dimensions of the box also need to be calculated carefully, not only because of low frequency problems, but also because the bigger the box, the longer it takes the software to calculate the field.

The typical way to feed an antenna in HFSS is to create a rectangular wave port and to connect the feeding cables that come from the antenna. In the case of a monopole antenna, a cable must be connected to the wave port and the monopole, and the wave port must also be connected to the electrical earth.

However, the size of the feeding cable from the wave port to the monopole must have relatively small dimensions compared to the dimensions of the monopole, in order to guarantee that the cable does not irradiate, or at least that its own radiation is small enough compared to that of the antenna.

However, as mentioned previously, the dimension of the cable cannot be too small because otherwise it will not operate at the frequency of $260 \mathrm{kHz}$. Placing the cable inside a shielding cylinder (e.g., copper) that avoids radiation is not possible because the shielding cylinder cannot be connected to the wave port or to the monopole, and radiation would leak through the unconnected extremes of the cylinder.

To overcome this problem, we avoided the feeding cable by putting the wave port under the monopole and connecting it directly to the monopole. In order to avoid the influence of the cable that connects the wave port to the electrical earth both the earth and the cable that connects to it were placed outside the radiation box.

Figure 4 shows the model created in HFSS to simulate the monopole antenna and its far field radiation pattern. As can be seen, the radiation pattern is omnidirectional, which is the origin of the interference problems of the theremin. 


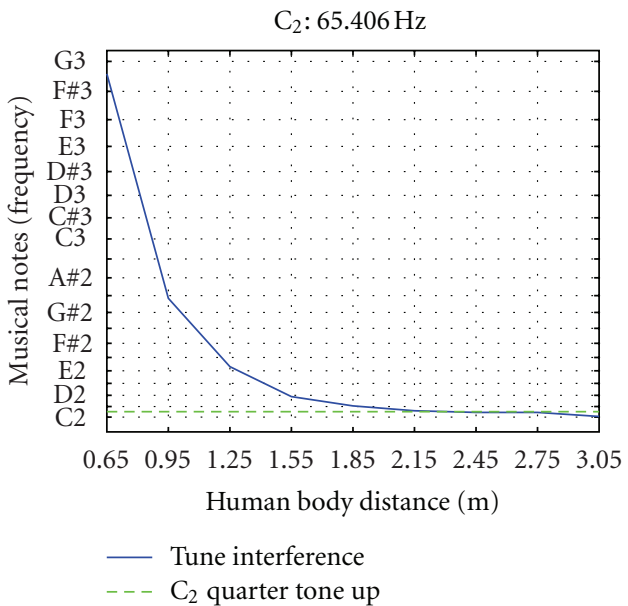

(a)

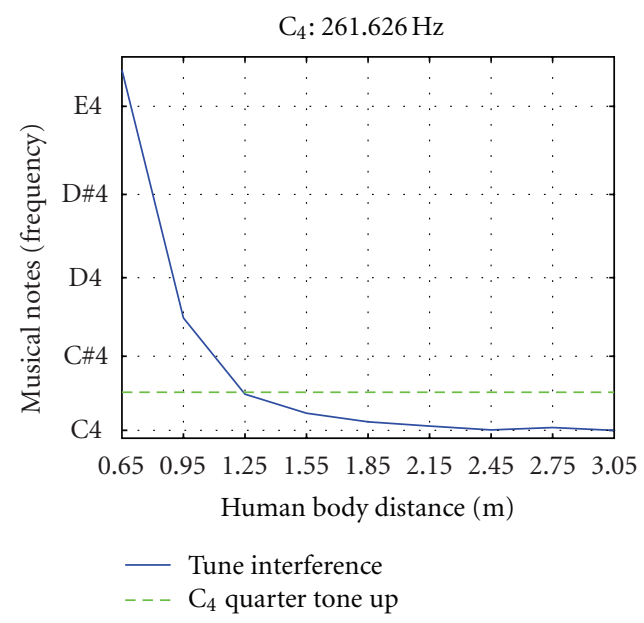

(c)

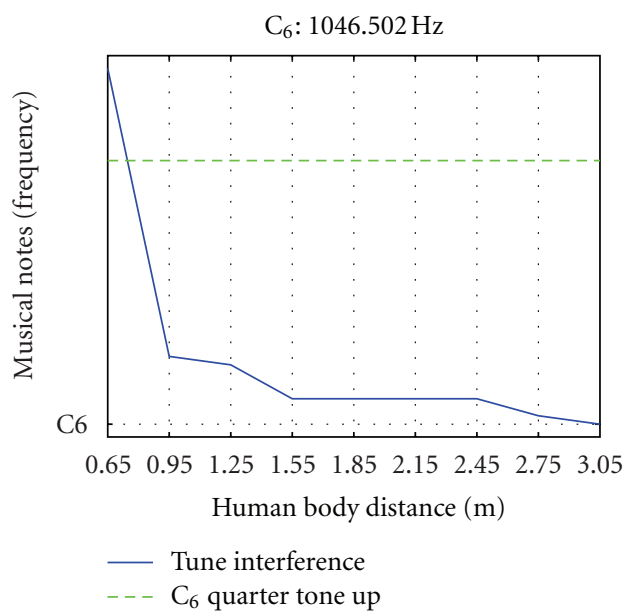

(e)

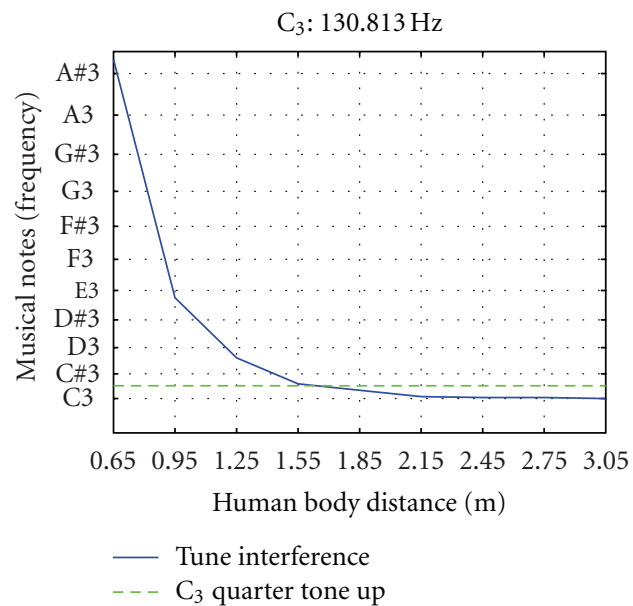

(b)

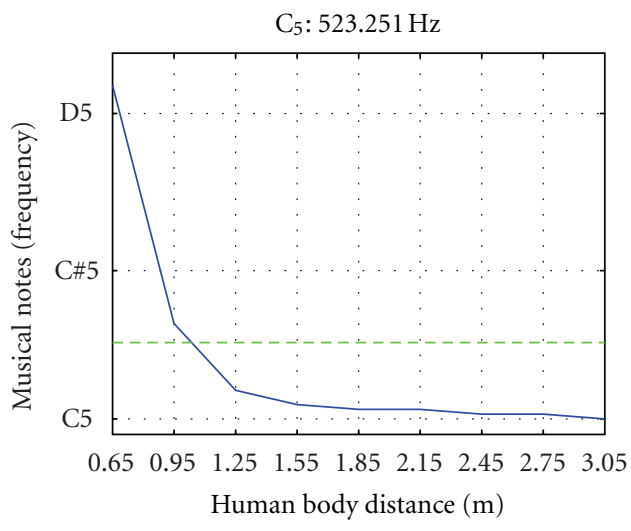

- Tune interference

- - $\mathrm{C}_{5}$ quarter tone up

(d)

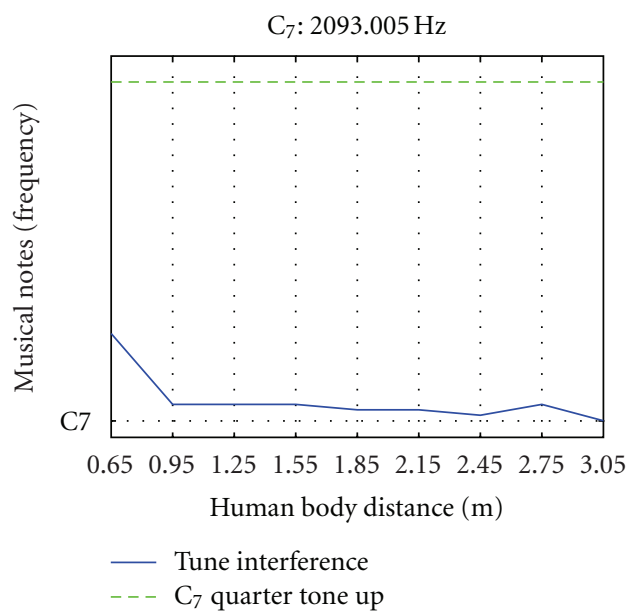

(f)

Figure 3: Results of human body interference in the theremin tune. 


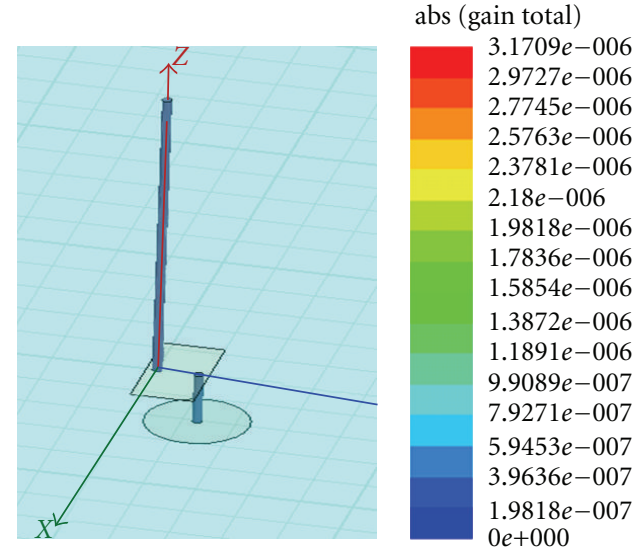

(a)

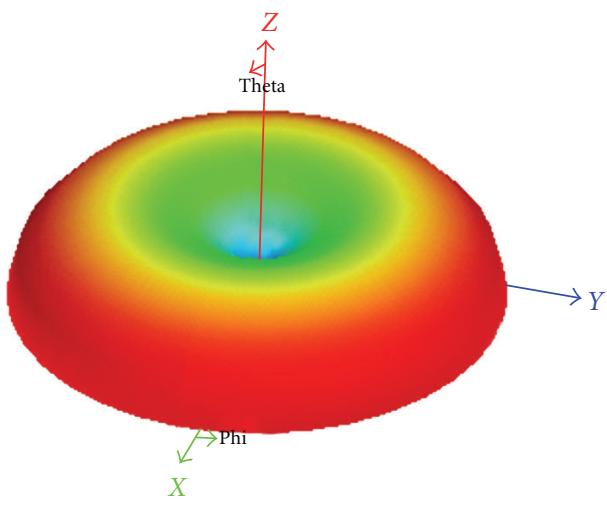

(b)

Figure 4: (a) Model of the monopole antenna in HFSS, (b) Far field radiation pattern of the monopole antenna.

The input impedance of the antenna was also simulated at different frequencies. At $260 \mathrm{KHz}$, the input reactance (imaginary part of the impedance) is $-\mathrm{j} 58353 \Omega$, very close to the estimated value obtained using real parameters of the theremin monopole and the theoretical formulas for the input impedance of a monopole, which gives $-\mathrm{j} 61213 \Omega$ [6]. This agreement proves the validity of the model used to simulate the antenna.

5.2. Isolation with Parasitic Metallic Bars. The HFSS model is going to be used to simulate the effect of an array of parasitic metallic bars which are placed in order to isolate the monopole antenna and to reduce the interference from a nearby person. The interfering person is modeled in HFSS by a cylinder of sea water (humans are composed of around $75 \%$ water) with a height of $900 \mathrm{~mm}$ (distance from head to waist since the rest of the body is out of the radiation box) and a radius of $200 \mathrm{~mm}$.

When the interfering body is placed at a distance of approximately $1.7 \mathrm{~m}$, the input reactance is greatly changed. Its value at $260 \mathrm{kHz}$ is $-\mathrm{j} 1413230 \Omega$. This change in the reactance while the instrument is being played supposes great changes in the intonation of the output sound of the theremin.

Now we add the antenna array around the monopole, see Figure 5, to assess what happens to the imaginary part of the impedance entry. The design of the set of monopoles placed in a semicircle around the monopole of theremin was chosen to assure that their presence would not make significant change to the impedance entry of the instrument. The array of antennas is formed by 6 monopoles with a height of $450 \mathrm{~mm}$ and a square cross section of $20 \mathrm{~mm}$ of side length.

The array monopoles are placed $500 \mathrm{~mm}$ away from the theremin monopole and there is an angle of $40^{\circ}$ between each pair of array monopoles. Following the simulation, we achieved the results shown in Figure 5. It can be observed that the presence of the array of antennas push the radiation field towards the direction where the hand of the musician would be.

As regards to the impedance, the imaginary part is $-77428 \Omega$. That would change the resonance frequency and the pitch of the output sound with regard to the case when no shielding antenna array is placed. Nevertheless, the theremin has a rod that allows, in cases of variations of small significance, to compensate impedance shifts and adjust the resonant frequency and the pitch to their original values.

The size and position of the array elements have been chosen to ensure that, when simulating the model made up of monopole plus obstacle plus antenna array, the imaginary part of the impedance does not change with regard to the case when the obstacle is not present, that is, $-77428 \Omega$.

Nevertheless, the simulations do not model perfectly the behavior of the real antenna, so we made a real prototype of the antenna array and tested its utility and effectiveness.

5.3. Experiments. Figure 6 illustrates the setup used to measure the isolation provided by the antenna array. To this purpose, we have connected the instrument to an oscilloscope and recorded the frequency variations when an obstacle is placed near the system.

The array is formed by monopoles of aluminium $45 \mathrm{~cm}$ tall and a square cross-section of $20 \mathrm{~mm}$ of side length. The array monopoles, the theremin, and its monopole are all placed at the same level laying on a wooden support (see Figure $7(\mathrm{a})$ ). The measurements were done in an area big enough not to have other objects which could interfere.

From the beginning readings, were taken from the theremin $(899 \mathrm{~Hz}$ with the array present and $834 \mathrm{~Hz}$ without), without any obstacles around (except a metal bar in the vicinity of the monopole simulating the hand of a musician). Then, the frequency of the instrument was measured with a nearby obstacle, in this case a person, at different distances (with and without the array). The audio outlet of the theremin was connected to the oscilloscope 


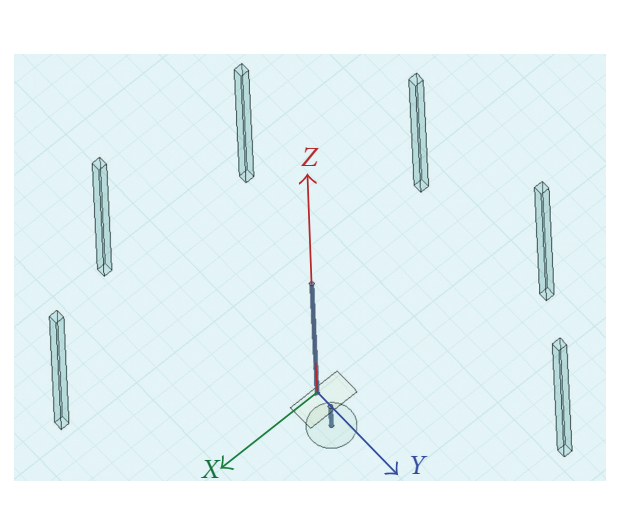

(a)

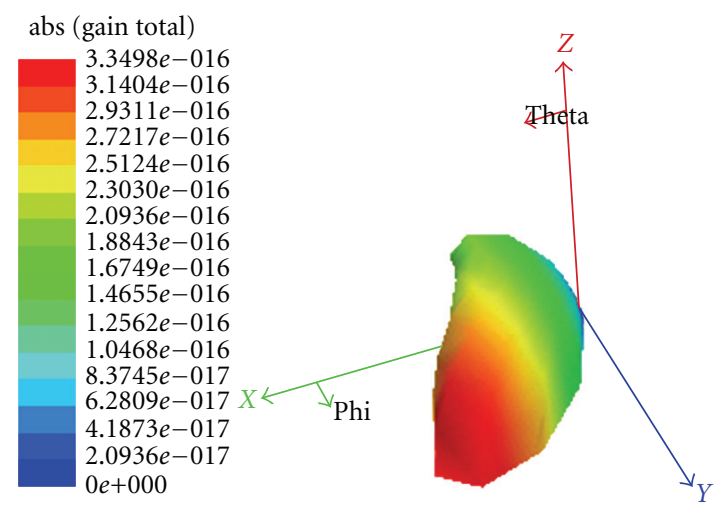

(b)

Figure 5: (a) Model of monopole + array of antenna, (b) far field radiation pattern.

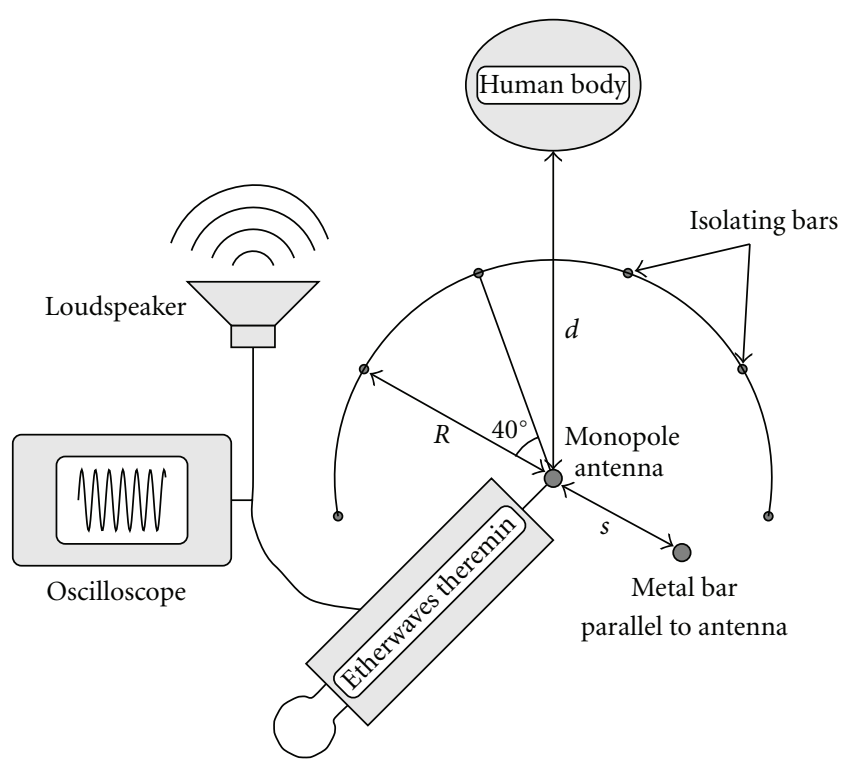

FIgURE 6: Schematic view.

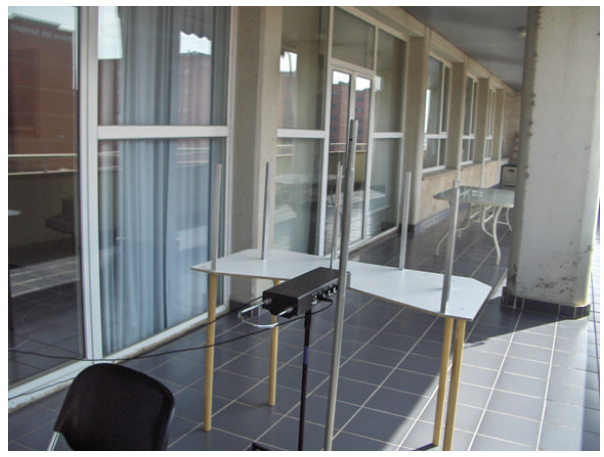

(a)

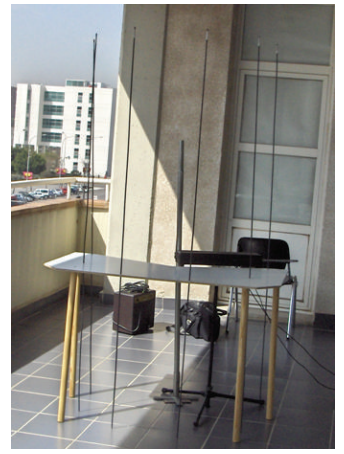

(b)

FIgURE 7: Measurement setup layout with isolating bars: (a) $45 \mathrm{~cm}$ isolating bars, (b) $2 \mathrm{~m}$ isolating bars. 


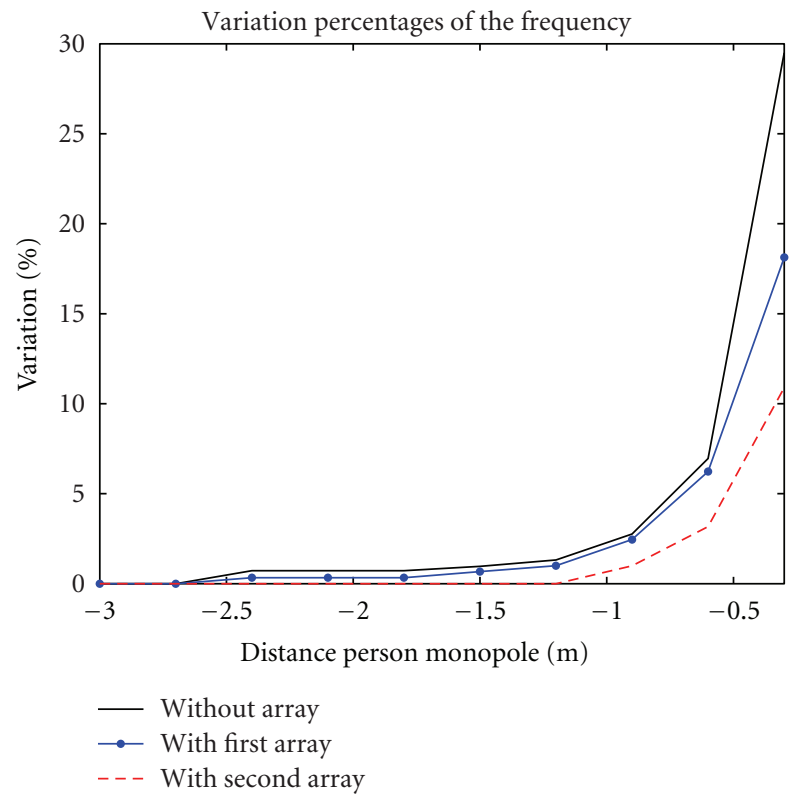

FIGURE 8: Curve showing the influence of a person upon the frequency of the theremin (variation percentages) without the array (straight curve), with the array of monopoles of $45 \mathrm{~cm}$ (dotted curve) and with the array of monopoles of $2 \mathrm{~m}$ (broken curve). The $\mathrm{C}_{5}$ note is played.

through which were seen the variations in frequency. The frequency variations were different to that extracted from the simulations. This is because in the simulation, the effect of what was under the level of the monopole (the legs of the human body) was not considered and in near field they also interfere.

After analyzing the results taken from the first experiment, we built a new array, using the original construction, but with metallic cylindrical monopoles $2 \mathrm{~m}$ high with a diameter of $6 \mathrm{~mm}$ (see Figure $7(\mathrm{~b})$ ). The new monopoles were inserted into the holes where the previous monopoles had been screwed into the wooden surface after the previous ones were removed. The acquired readings from the previous experiments can be seen in Figure 8.

Figure 8 clearly shows the increase (in percentage) of the frequency variation of the theremin little by little as the obstacle gets closer to the instrument.

The fact that the dotted curve (relating to the presence of the first set of arrays built) is found underneath the straight curve indicates the isolation effect (even if partial) achieved with the introduction of the array. In consequence, because the broken curve (relating to the second set built) is found underneath the dotted curve (and therefore also underneath the straight curve), this indicates that the isolation obtained thanks to the second array is much greater than that obtained with the first. Figure 9 presents the frequency variation in the same terms as Figure 3. As it happens without the isolating antenna array in the higher-pitched notes, the musician's hand is so near the theremin antenna that the interference effect is quite small and only affects if the interfering human body is really very close to the antenna.

In $\mathrm{C}_{7}$, there is so little interference that the small variations seem to be due only to tolerances in the measurement procedure. However, in $\mathrm{C}_{6}$ with the isolating array the out of tuning remains far lower a quarter tone, and that is not the case without isolation (see Figure 3).

In the middle-pitched area, the interference produces a higher tune of a quarter tone in the original note for distances as near as approximately $1.161 \mathrm{~m}$ for $\mathrm{C}_{4}$ and $0.986 \mathrm{~m}$ for $\mathrm{C}_{5}$, lower than the same distances without isolation, that is, $1.249 \mathrm{~m}$ for $\mathrm{C}_{4}$ and $1.05 \mathrm{~m}$ for $\mathrm{C}_{5}$.

Therefore, the interfering human body produces lower out of tuning at the same distance with the isolation bars. Finally, for the lower-pitched area those distances increase to approximately $1.5068 \mathrm{~m}$ for $\mathrm{C}_{3}$ and $2.054 \mathrm{~m}$ for $\mathrm{C}_{2}$, because of the greater distance of the musician hand to the antenna to produce these notes and the lower frequency differences between musical notes in that area. Note that those distances where $1.665 \mathrm{~m}$ for $\mathrm{C}_{3}$ and $2.359 \mathrm{~m}$ for $\mathrm{C}_{2}$.

\section{Conclusions}

To conclude, the assessment done shows how, at a musical level, the array proved to bring an overall improvement for the interferences, since the variations provoked by an obstacle approaching the instrument are smaller, and it has to be nearer than before to produce the same out of tuning.

Nevertheless, due to the decreasing frequency difference between musical notes at the lower octaves, and the greater musician hand distance to the theremin antenna, the isolation in octaves 2 and 3 is not enough for a satisfactory performance. Different solutions are possible for better isolating the electromagnetic field at those frequencies such as considering larger array monopoles or building a metallic "roof" over the theremin antenna, approaching more to a Faraday jail.

Our future work is considering both solutions, taking into account that both of them will also improve the 


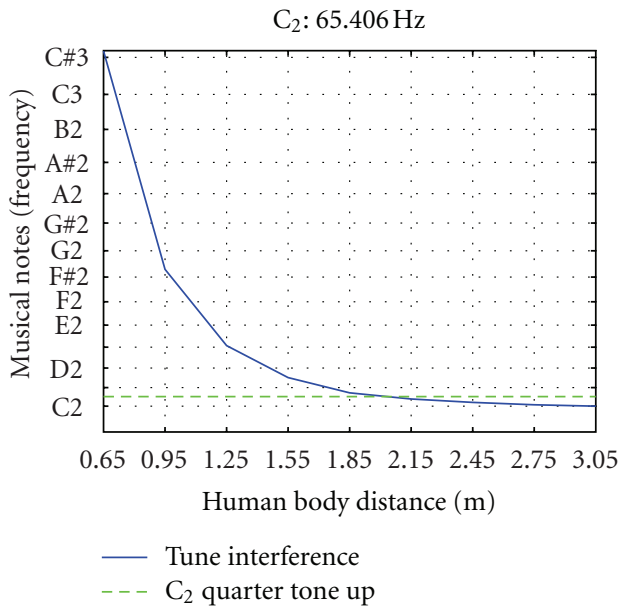

(a)

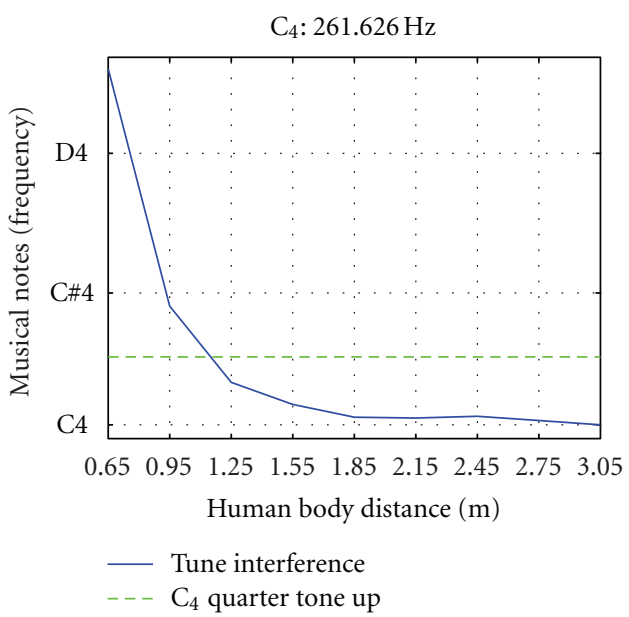

(c)

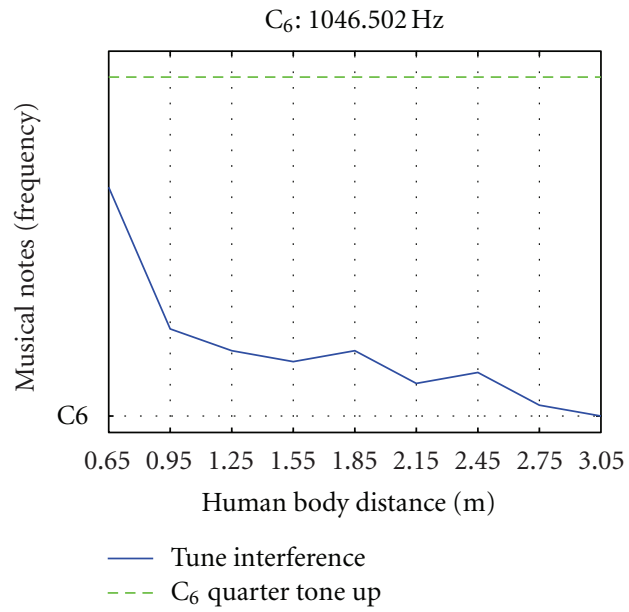

(e)

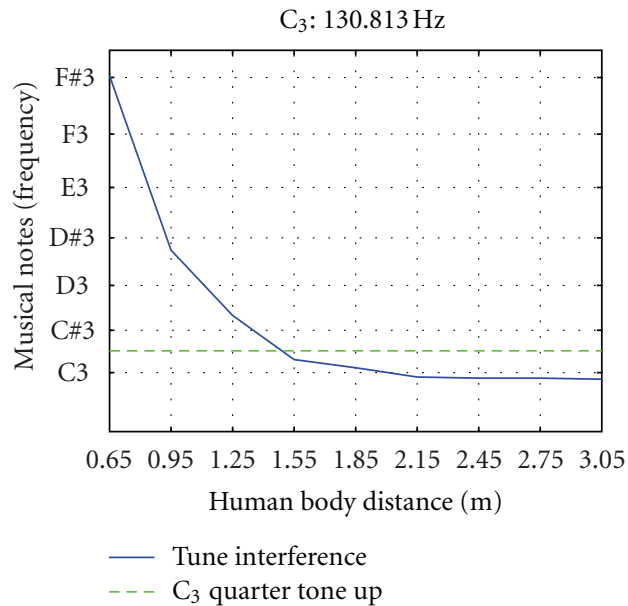

(b)

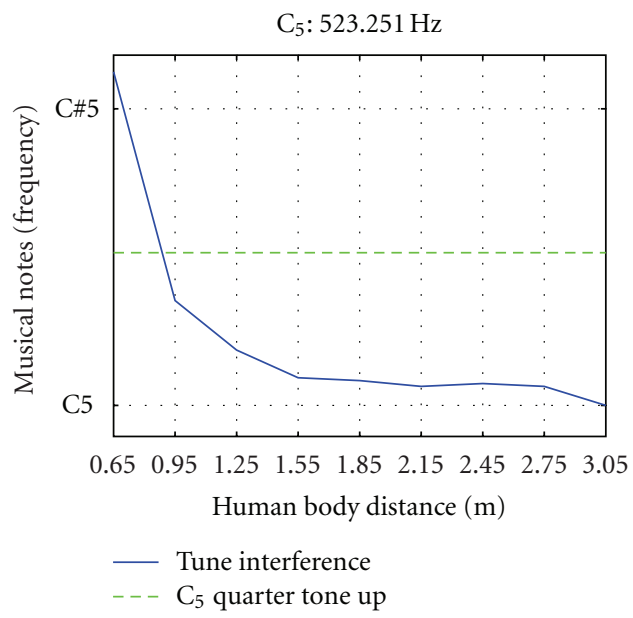

(d)

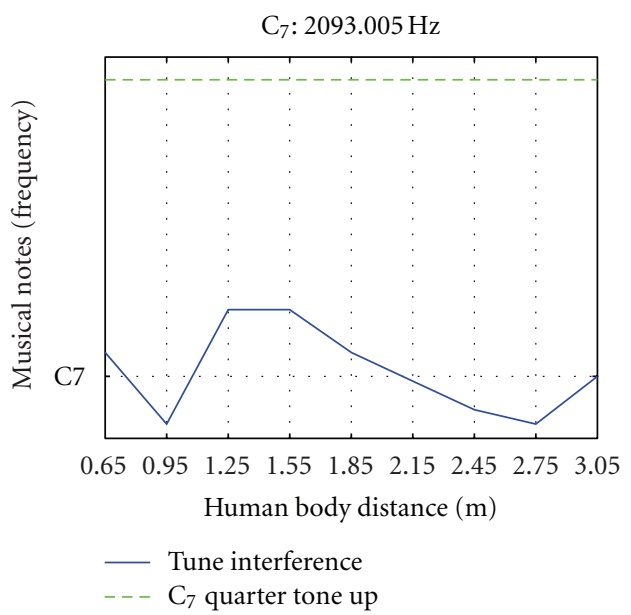

(f)

FIGURE 9: Results of human body interference in the theremin tune with isolating antenna array $2 \mathrm{~m}$ tall. 
performance at the rest of frequencies. The next step will be to get the players' opinion about the possibility of a live performance surrounded by the vertical array, in order to connect the obtained results with real applications.

\section{References}

[1] A. Glinsky, Theremin: Ether Music and Espionage, University of Illinois Press, 2000.

[2] “Theremin World," 2011, http://www.thereminworld.com/.

[3] Thierry Frenkel, "Theremin related research, design, modifications and repair," 2011, http://theremin.tfrenkel.com/.

[4] W. Buller and B. Wilson, "Measurement and modeling mutual capacitance of electrical wiring and humans," IEEE Transactions on Instrumentation and Measurement, vol. 55, no. 5, pp. 1519 $1522,2006$.

[5] C. Bachiller, C. Hernández, and J. Sastre, "Collaborative Learning, Research and Science Promotion in a Multidisciplinary Scenario: information and Communications Technology and Music," in Proceedings of the International Conference on Engineering Education (ICEE '10), Gliwice, Poland, 2010.

[6] C. Balanis, Antenna Theory: Analysis and Design, John Wiley \& Sons, 1997. 

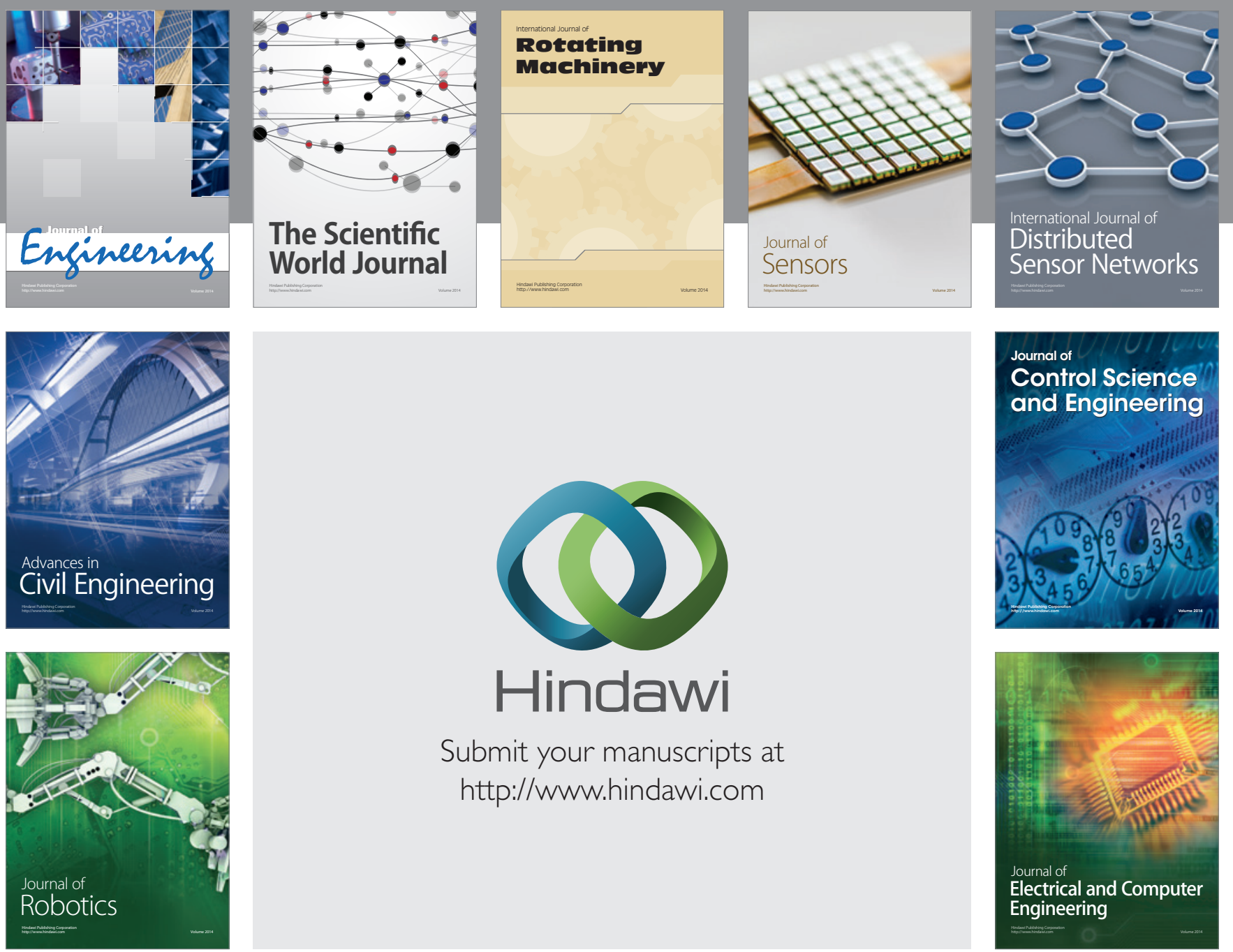

Submit your manuscripts at

http://www.hindawi.com
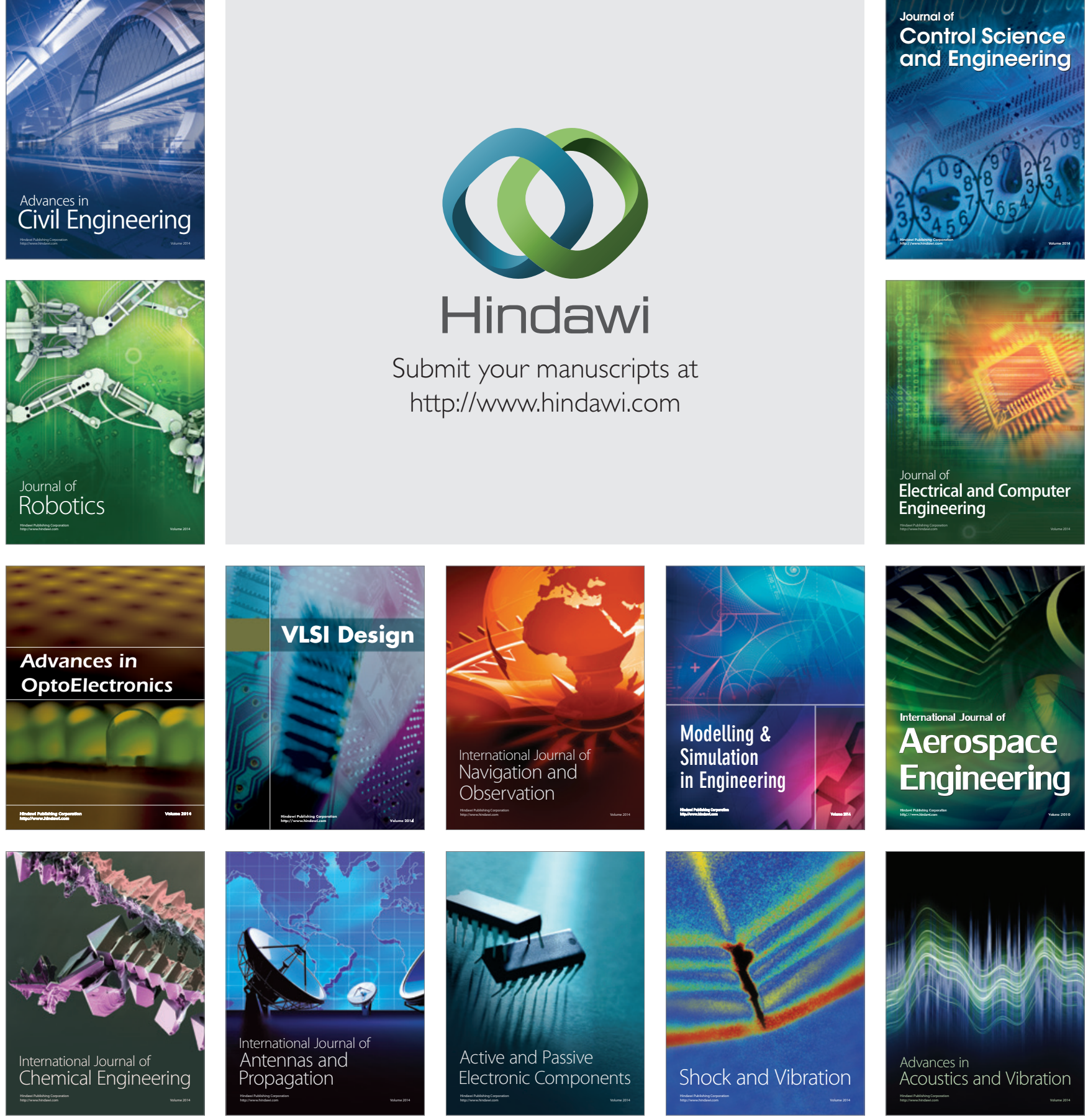